\title{
Abitudini ed ereditarietà: la rivincita di Lamarck?
}

\author{
Alessandro Capitanini ${ }^{1}$, Francesca Capitanini ${ }^{2}$ \\ ${ }^{1}$ SOC Nefrologia e Dialisi Ospedale San Jacopo, Pistoia, e ASL Toscana Centro - Italy \\ ${ }^{2}$ Facoltà di Medicina Università degli Studi di Firenze, Firenze - Italy
}

\begin{abstract}
Habits and genetic inheritance: Lamarck's revenge?
Human phenotype, the set of characteristics manifested by a living organism, is determined by genetic information expression dependent on genome, epigenome and microbiome. There is a kind of bidirectionality between humans and their genome with significant influence by environment and human behaviour. In the timeline of evolution we see that genetic modifications take millions of years to take place and consolidate, as per Darwinian principles, but environment and our habits are able much more rapidly to influence our phenotypic response, through epigenetic and microbiotic pathways, as per Lamarckian hypothesis (Fig. 1). Our habits (physical, psychological, environment) are able to determine changes in gene expression and potentially influence our children's one. The evidence of these new concepts should be a further stimulus to a more conscious lifestyle.
\end{abstract}

Keywords: Epigenetic inheritance, Epigenome, Genome, Habits, Microbiome

Fin dagli albori della vita sulla terra, gli uomini si sono interrogati sulle loro origini, sull'origine degli animali e del mondo intero. I grandi pensatori dell'antica Grecia hanno prodotto tante delle più suggestive e affascinanti teorie. Nella fattispecie, per quanto riguarda la biologia, spicca quella di Aristotele, formulata durante il XV secolo a.C: il Fissismo. Secondo la concezione aristotelica, le specie vegetali e animali sono destinate a rimanere sempre uguali a loro stesse, statiche e immutabili, poiché create con scopi e caratteristiche ben precisi.

Tale ipotesi ha predominato per secoli, trovando l'appoggio di nuovi studiosi e pensatori europei, fino agli studi di Charles Darwin, autore dell'opera "L'origine delle specie per selezione naturale", pubblicata nel 1859, e in seguito all'accertamento dell'ereditarietà genetica. Fu un vero e proprio punto di svolta, in grado di cambiare per secoli l'orientamento scientifico in merito e di condannare, quasi ridicolizzandole, tutte le altre ipotesi fino ad allora formulate.

Tra queste, quella sull'evoluzione basata su adattamento ed ereditarietà dei caratteri acquisiti di Jean-Baptiste Lamarck, naturalista e zoologo francese. Egli spiegò la sua teoria con l'emblematico esempio del collo delle giraffe: secondo lo studioso, in origine, esistevano solo giraffe con il collo corto ma

Received: June 29, 2021

Accepted: July 1, 2021

Published online: July 19, 2021

Indirizzo per la corrispondenza:

Alessandro Capitanini

SOC Nefrologia Pistoia

Via ciliegiole

51100 Pistoia - Italy

drcapitanini@gmail.com nel tempo, grazie agli sforzi operati per procacciarsi cibo dai rami più alti, i vari esemplari avrebbero sviluppato la muscolatura delle zampe anteriori e allungato il collo, trasmettendo, il carattere, appunto acquisito, alla prole.

In effetti, uno dei cardini della genetica classica è rappresentato dal concetto di eredità fedele dei genotipi da una generazione all'altra, mediata dalla copia e dalla trasmissione del DNA. Tuttavia, oltre all'ereditarietà del genoma, sta emergendo in modo sempre più inconfutabile che il mantenimento del destino cellulare dipende anche dall'ereditarietà delle informazioni epigenetiche (1). II DNA, dunque, non è l'unica informazione genetica a essere tramandata dai genitori ai figli (2).

Uno studio pubblicato su Science (3) dai ricercatori del Max-Planck-Institut per l'immunobiologia e l'epigenetica a Friburgo, in Germania, ha raccolto prove rigorose del fatto che anche le istruzioni epigenetiche, modifiche che all'interno delle cellule regolano l'espressione dei geni senza che sia alterata la sequenza del DNA, sono trasmesse alla prole. La conclusione è di grande rilievo perché mette fine a un dibattito durato molti decenni: nella visione tradizionale le modificazioni epigenetiche, dovute all'interazione dell'individuo con l'ambiente che lo circonda (compresi, per esempio, i suoi stili di vita e la sua alimentazione), non possono oltrepassare il confine tra le generazioni, come se venissero azzerate durante lo sviluppo dello spermatozoo e della cellula uovo. Nella concezione più moderna questo risulta invece possibile.

L'idea di una possibile ereditarietà intergenerazionale delle regolazioni epigenetiche è stata infatti ipotizzata nei primi anni novanta: alcuni importanti studi epidemiologici hanno mostrato, per esempio, una forte correlazione tra l'aumento di assunzione di cibo di un soggetto e un maggior rischio di diabete e di malattie cardiovascolari nei nipoti. 
lovino et al., nel lavoro pubblicato da Science, hanno analizzato l'ereditarietà epigenetica del moscerino della frutta (Drosophila melanogaster), insetto molto studiato in biologia per la sua semplicità. II gruppo si è concentrato in particolare su una modifica chiamata H3K27me3, associata alla soppressione dell'espressione genica. H3K27me3 si può trovare anche negli esseri umani e ha, appunto, la funzione di alterare la cromatina, modificando l'accesso alla catena del DNA. II risultato più rilevante dello studio è stato quello di aver documentato che le modifiche H3K27me3 che contrassegnavano la cromatina erano ancora presenti negli embrioni dopo la fecondazione, anche se altri marcatori epigenetici erano stati cancellati. Ciò indica che la madre può passare almeno parte dei suoi marcatori epigenetici alla prole.

Sebbene la sequenza del DNA sia la responsabile della maggior parte dell'ereditarietà, comprese alcune malattie, sta diventando chiaro che anche l'ereditarietà epigenetica può avere un ruolo non trascurabile (4-7).

Tutto questo risulta indubbiamente rivoluzionario da un punto di vista scientifico ma, sorprendentemente, anche da un punto di vista etico e morale: l'individuo deve rendersi consapevole del fatto che le proprie abitudini possono influenzare non solo il proprio destino ma anche quello della propria prole.

Una delle abitudini che ha un peso notevole sulle sorti della salute dell'uomo è la sedentarietà. Anche la sedentarietà è, almeno in parte, frutto dell'espressione genica, come dimostrano gli studi su coppie di gemelli (8) e diversi lavori sperimentali animali (9). Ma come è possibile che l'evoluzione prenda la strada sbagliata? Perché le persone tendono a evitare l'esercizio nonostante i suoi benefici?

Secondo l'opinione dell'antropologo Lieberman (10), il limitato accesso al cibo, dunque alle calorie, dell'uomo cacciatore/raccoglitore avrebbe guidato a un comportamento istintivo di ricerca del riposo durante il giorno per evitare un inutile dispendio energetico. Purtroppo, come sottolinea Lieberman, l'evoluzione non ha mai avuto l'opportunità di sviluppare protezioni contro l'inattività fisica, che quindi, se inappropriata, non induce, almeno per ora, strategie difensive dell'organismo.

Se è vero che il genoma la fa da padrone, si rende oggi evidente che l'ambiente e le nostre abitudini sono in grado di influire sul nostro fenotipo in modo determinante, in tempi relativamente rapidi. Studi condotti sulla popolazione degli indiani Pima, una popolazione fortemente predisposta a obesità e diabete mellito di tipo 2 (T2D), ci portano a considerazioni molto suggestive. Nella storia di questo particolare popolo, circa 1.000 anni fa, si è verificata una separazione: parte dei Pima rimase in Messico e parte migrò in Arizona. Pur condividendo un background genetico comune, ad oggi queste due popolazioni mostrano notevoli differenze, soprattutto in merito al rischio di T2D. I Pima che risiedono in Arizona hanno adottato uno stile di vita occidentale, caratterizzato da inattività fisica e alimentazione eccessiva e oggi, presentano tra le più alte prevalenze al mondo di T2D (11). Al contrario, la popolazione indiana Pima messicana ha mantenuto la sua prevalenza storica, relativamente bassa, di T2D (12), correlata a uno stile di vita fisicamente attivo caratterizzato da un'agricoltura non meccanizzata, allevamento del bestiame e lavoro domestico. Per quantificare queste differenze comportamentali: si stima che gli indiani Pima dell'Arizona assumano $\sim 500-600 \mathrm{kcal} /$ giorno in più rispetto ai loro omologhi messicani (13). Sebbene le sequenze del DNA, con molta probabilità, non siano cambiate durante la separazione di 1.000 anni, è piuttosto probabile che si siano verificati cambiamenti epigenetici nella popolazione Pima dell'Arizona a causa dei cambiamenti dello stile di vita (14).

Le certezze della Genetica, così come l'abbiamo appresa nel corso dei nostri studi universitari, evidenziano crepe sempre più profonde e non ignorabili, come sostiene il Prof. Mattick nel suo editoriale dal titolo "rocking the foundations of molecular genetics" (15).

Ma non è tutto: proprio in questi ultimi anni stiamo scoprendo che non solo il genoma e l'epigenoma, bensì anche il microbioma (patrimonio genetico dei microrganismi che popolano il corpo umano), contribuiscono a definire la nostra identità biologica e fenotipica.

Il concetto di microbioma si deve a Joshua Lederberg, premio Nobel per la medicina nel 1958 (16). Secondo Lederberg, il Progetto Genoma Umano (progetto di ricerca scientifica internazionale il cui obiettivo principale era quello di identificare e mappare i geni del genoma umano) avrebbe dovuto comprendere anche lo studio dei microrganismi ospitati nella specie umana, il cui insieme può essere paragonato a un vero e proprio organo supplementare, in virtù delle funzioni fondamentali da esso svolte $(17,18)$. Due ricerche, pubblicate recentemente in uno speciale su Science (19), hanno evidenziato le importanti influenze di ambiente e abitudini su selezione ed espressione del genoma microbico, che, a sua volta, condiziona la salute del proprio ospite.

In conclusione, II fenotipo umano, ossia l'insieme delle caratteristiche manifestate da un organismo vivente, viene determinato dall'espressione di informazioni genetiche dipendenti dal genoma, dall'epigenoma e dal microbioma. Vige una sorta di doppio senso tra l'influenza sull'uomo da parte del suo genoma e l'influenza sul genoma stesso da parte del comportamento umano e dell'ambiente.

Nella timeline dell'evoluzione vediamo che le modificazioni genetiche impiegano milioni di anni per realizzarsi e consolidarsi, come da principi darwiniani, ma l'ambiente e le nostre abitudini sono in grado, molto più rapidamente, di influenzare la nostra risposta fenotipica, in termini epigenetici e microbiotici, in un certo senso come da pensiero lamarckiano (Fig. 1). Con le nostre abitudini, sfera fisica, psichica e ambientale, siamo in grado di determinare modifiche dell'espressione genica e, potenzialmente, di influenzare anche quelle dei nostri figli. L'evidenza di questi nuovi concetti deve essere un ulteriore stimolo a vivere più consapevolmente. 


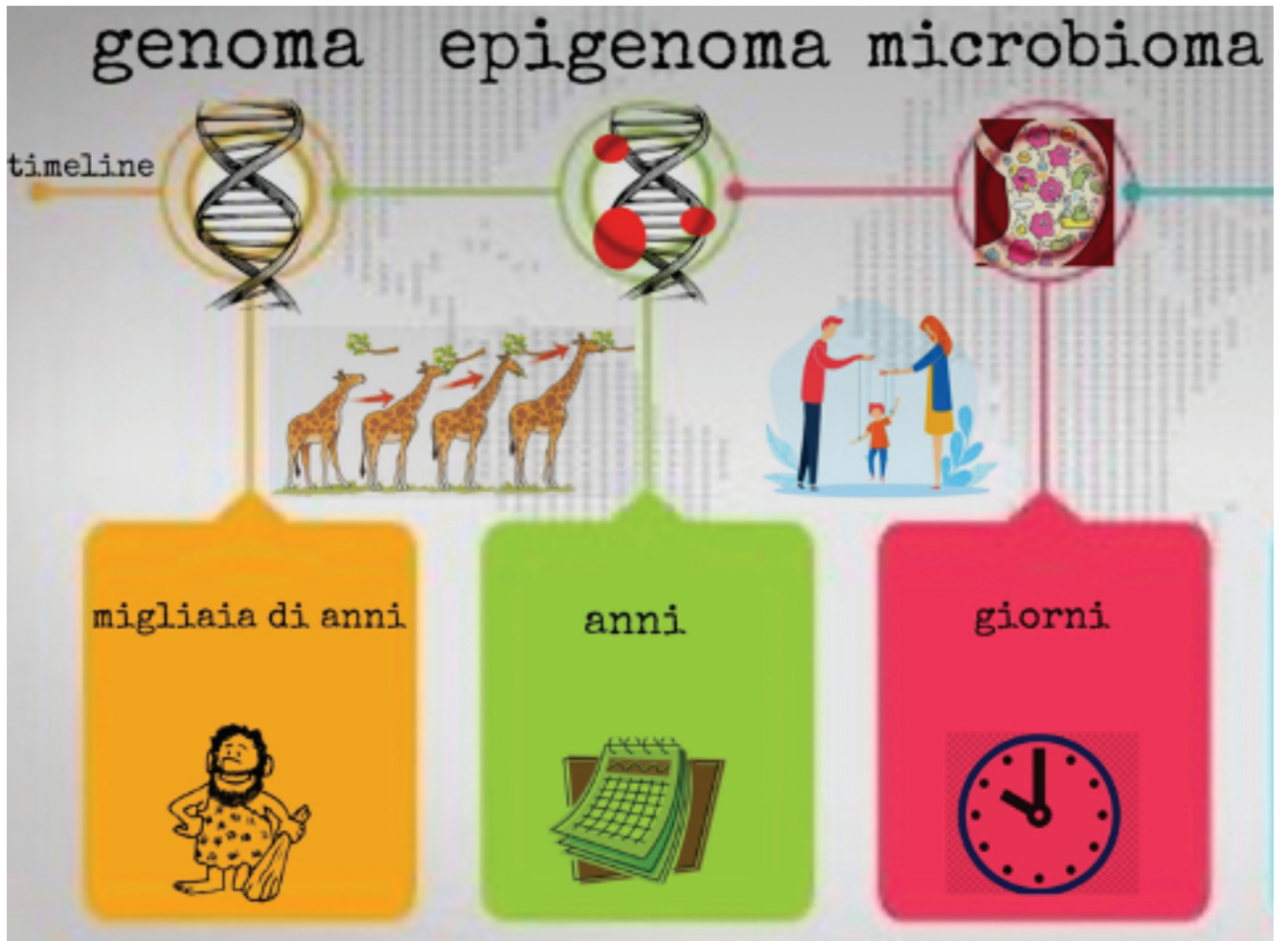

Fig. 1 - Timeline delle mutazioni genomiche, epigenomiche e microbiomiche. La giraffa, simbolo delle teorie di Lamarck. La possibile trasmissione verticale (dai genitori ai figli) di alcune caratteristiche epigenetiche.

\section{Disclosures}

Conflict of interest: The authors declare no conflict of interest. Financial support: This research received no specific grant from any funding agency in the public, commercial, or not-for-profit sectors. Authors' contribution: All authors contributed equally to this manuscript.

\section{Bibliografia}

1. Gurdon JB. From nuclear transfer to nuclear reprogramming: the reversal of cell differentiation. Annu Rev Cell Dev Biol. 2006;22(1):1-22. CrossRef PubMed

2. Lambrot R, Xu C, Saint-Phar S, et al. Low paternal dietary folate alters the mouse sperm epigenome and is associated with negative pregnancy outcomes. Nat Commun. 2013;4(1):2889. CrossRef PubMed

3. Zenk F, Loeser E, Schiavo R, Kilpert F, Bogdanović O, lovino N. Germ line-inherited H3K27me3 restricts enhancer function during maternal-to-zygotic transition. Science. 2017;357(6347): 212-216. CrossRef PubMed

4. Jirtle RL, Skinner MK. Environmental epigenomics and disease susceptibility. Nat Rev Genet. 2007;8(4):253-262. CrossRef PubMed

5. Tang $W-Y$, Ho SM. Epigenetic reprogramming and imprinting in origins of disease. Rev Endocr Metab Disord. 2007;8(2):173182. CrossRef PubMed

6. de Assis S, Warri A, Cruz MI, et al. High-fat or ethinyl-oestradiol intake during pregnancy increases mammary cancer risk in several generations of offspring. Nat Commun. 2012;3(1):1053. CrossRef PubMed

7. Gapp K, Jawaid A, Sarkies P, et al. Implication of sperm RNAs in transgenerational inheritance of the effects of early trauma in mice. Nat Neurosci. 2014;17(5):667-669. CrossRef PubMed

8. Roberts MD, Brown JD, Company JM, et al. Phenotypic and molecular differences between rats selectively bred to voluntarily run high vs. low nightly distances. Am J Physiol Regul Integr Comp Physiol. 2013;304(11):R1024-R1035. CrossRef PubMed

9. den Hoed M, Brage $\mathrm{S}$, Zhao JH, et al. Heritability of objectively assessed daily physical activity and sedentary behavior. Am J Clin Nutr. 2013;98(5):1317-1325. CrossRef PubMed

10. Lieberman DE. Is exercise really medicine? an evolutionary perspective. Curr Sports Med Rep. 2015;14(4):313-319. CrossRef PubMed

11. Schulz LO, Bennett PH, Ravussin E, et al. Effects of traditional and western environments on prevalence of type 2 diabetes in Pima Indians in Mexico and the U.S. Diabetes Care. 2006;29(8):1866-1871. CrossRef PubMed

12. Ravussin E, Valencia ME, Esparza J, Bennett PH, Schulz LO. Effects of a traditional lifestyle on obesity in Pima Indians. Diabetes Care. 1994;17(9):1067-1074. CrossRef PubMed

13. Esparza J, Fox C, Harper IT, et al. Daily energy expenditure in Mexican and USA Pima indians: low physical activity as a possible cause of obesity. Int J Obes. 2000;24(1):55-59. CrossRef PubMed

14. Noble D. Conrad Waddington and the origin of epigenetics. $J$ Exp Biol. 2015;218(Pt 6):816-818. CrossRef PubMed

15. Mattick JS. Rocking the foundations of molecular genetics. Proc Natl Acad Sci USA. 2012;109(41):16400-16401. CrossRef PubMed

16. Lederberg J. The microbe's contribution to biology-50 years after. Int Microbiol. 2006;9(3):155-156. PubMed

17. Burcelin $R$, Luche $E$, Serino $M$, Amar J. The gut microbiota ecology: a new opportunity for the treatment of metabolic diseases? Front Biosci. 2009;14(1):5107-5117. CrossRef PubMed

18. Mans JJ, von Lackum K, Dorsey $C$, et al. The degree of microbiome complexity influences the epithelial response to infection. BMC Genomics. 2009;10(1):380. CrossRef PubMed

19. Andrew H. Moeller, Taichi A. Suzuki, Megan Phifer-Rixer, Michael W. Nacham. Trasmission mode of mammalian gut. Science. 2018;26(oct):453-457. 